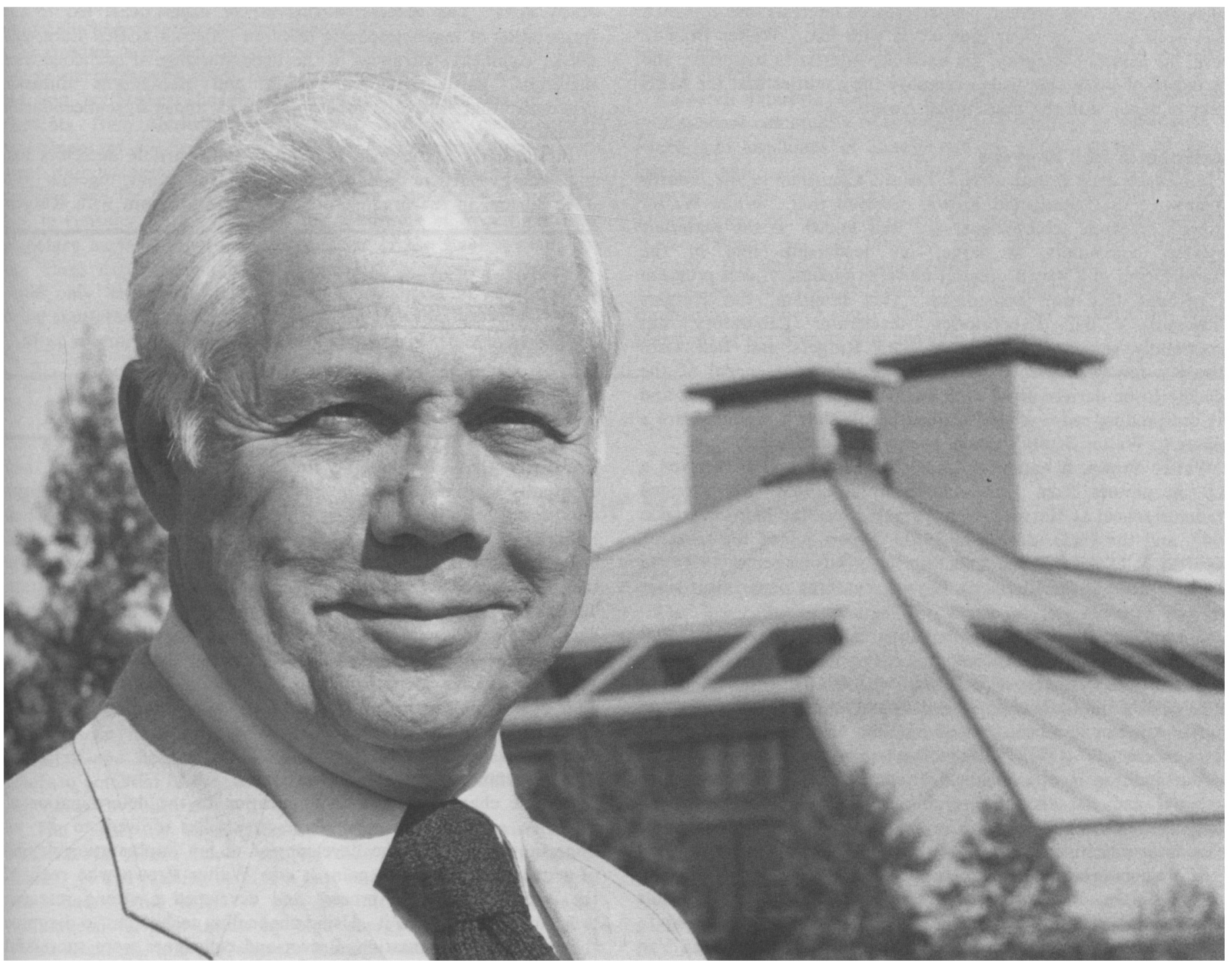

WALTER BROWN

\title{
Von Hippel Winner
}

\section{Materials science's highest honor Accorded Bell Labs' Walter Brown}

The Materials Research Society is most pleased to recognize Walter L. Brown, a distinguished research leader, with its 1984 Arthur Von Hippel Award. The award, which consists of an embedded ruby crystal, a cash honorarium, and lifetime membership in the Society, will be presented at a ceremony at 6:30 p.m. Wednesday, Nov. 28, in the Grand Ballroom of the Boston Marriott/Copley Place Hotel as a featured item on the Society's annual meeting agenda.

Walter Brown is Head: Radiation Physics Research Department at AT\&T Bell Laboratories in Murray Hill, New Jersey. Brown, a member of the Materials Research Society, the American
Association for the Advancement of Science, and a Fellow of the American Physical Society, has established an extensive record of achievement in the field of semiconductor science and technology, and leadership in university-industry collaboration.

In announcing the award, Society President C.W. White said of Brown, "Walter Brown's technical achievements have had a truly global impact upon the world of both science and technology. His work on surface states, inversion layers, ion channeling, sputtering, and particle-solid interactions is at the core of a significant fraction of today's materials science. In addition, Brown's influence in the field of materials research is amplified by the large number of 
colleagues, past and present, whose research interests and approach have been shaped by their interaction with him. Walter Brown's work, his personal integrity, his unfailing interest in materials, and his record of leadership truly exemplify the qualities that the MRS seeks to honor with the Von Hippel Award."

\section{Leadership in Joint Programs}

Immediate Past President H.J. Leamy, Chairman of the Awards Committee that nominated Brown, observed that, "While Walter Brown's research achievements are well known to the materials research community at large, his leadership role in the establishment of a very successful university-industry joint program is perhaps less well recognized. This program, the Rutgers University - Bell Laboratories Accelerator Laboratory, has successfully served the needs of both Rutgers and Bell Labs scientists for over twenty years, and stands as a model of the benefits to be derived from such collaboration. This program and the outstanding cadre of well trained people that it produced are a tribute to Walter Brown's vision, energy, and dedication."

Walter Brown, a native of Charlottesville, Virginia, received a B.S. in physics from Duke University in 1945. He attended graduate school at Harvard, which granted him the M.A. degree in 1947, and the Ph.D. degree in 1951. Brown joined the research department of AT\&T Bell Laboratories in November of 1950 and has been head of the Radiation Physics Research department since 1958.

\section{The Basis of MOSFET Technology}

His early experiments at Bell Labs with Brattain, Shockley, and Fletcher laid the foundation of understanding of surface states and inversion layers in silicon and germanium that made possible the development of MOSFET technology. He used the variation of conductance with external field to determine both the surface potential and the distribution of charge in surface states, and recognized that observation of the unique minimum in conductance leads to quantitative results.

Brown's interest in semiconductors led to the design of solid state radiation detectors that were flown in the first Telstar experimental communications satellite, and which were used to determine the nature of the particles that are trapped in the Van
Allen belts. The success of this set of experiments led to the formulation of magnetospheric research program at Bell Labs, and netted significant advances in the understanding of semiconductor materials, particularly the boron and phosphorus diffusion processes that are so fundamental to today's semiconductor industry.

In the early Sixties, Brown's interest in particle detectors and high energy particle damage led to his involvement, together with J.A. Burton, in the organization of a joint program with Rutgers
Walter Brown's superior work, His personal integrity, and his Leadership exemplify the qualities The Von Hippel Award seeks to honor

University that would serve to provide shared equipment and eventually to stimulate the interest and dedicated efforts of a large number of materials scientists. The Rutgers University - Bell Laboratory Accelerator Laboratory and Walter Brown's research department at Bell Labs were at the center of a global interest in the phenomenon of particle channeling and blocking in crystals. The joint program resulted in the granting of a dozen Ph.D. degrees to graduate students whose research was directed wholly or in part by Bell Labs scientists. Among the alumni of the Accelerator Laboratory are many prominent materials scientists and MRS members-B.R. Appleton, L.C. Feldman, and J.K. Hirvonen, to name but three.

\section{The Importance of Particle Channeling}

Particle channeling and its application to the determination of impurity site location and damage studies proved to be an important adjunct to the development of ion implantation doping of semiconductors, and again, it was Walter Brown who realized the importance of the process and developed a strong research program to focus upon it. Using channeling techniques to diagnose the effects of implantation, Brown and colleagues were successful

\section{The Award}

The Materials Research Society's most prestigious award, the Von Hippel Award, is presented annually to the living scientist who, in the Society's estimation, best exemplifies the originality, brilliance of intellect and diligence of purpose throughout a career that are the hallmark of science at its best; who adheres to the strictest ethical standards and highest code of professional integrity, and whose vision transcends conventional disciplines, as does the science of materials itself.

The award consists of a synthetic ruby crystal suitably mounted, a cash honorarium, and lifetime membership in the Society. It is named for Arthur Robert von Hippel, emeritus professor of the Massachusetts Institute of Technology, who was also its first recipient.

Annually, the Awards Committee of the MRS Council solicits nominations for the Von Hippel Award from among the membership and elsewhere in the community of materials scientists. The nominees are evaluated against the Society's criteria, rather than against each other; i.e., the award is not presented in any year in which a sufficiently outstanding scientist is not put forward. Contrariwise, only a single individual can receive the prize in any year; the recipient could be chosen from among a group of distinguished scientists at least some of whom are destined to receive the award in subsequent years.

From among the candidates it has reviewed, the Awards Committee puts forward the names of the principal contenders for the honor. It reserves the right to nominate no one should the men and women it considers lack the requisite professional stature. The nominees are considered in confidence by the executive committee and council of the Society. This body, representative of the many disciplines and institutions within the MRS, make the final decision by means of a majority vote. 
in generating techniques for the successful use of implantation in the production of semiconductor devices.

Sputtering, a process also observed during the interaction of high energy particles with solids, also captured Walter Brown's interest. He and colleagues showed the preferential sputtering of light elements from multicomponent solids, and the consequential enrichment of the surface with heavier elements-a discovery of great significance when sputtering is contemplated as a depth profiling tool for chemical analysis. Brown's sputtering interest led him to consider, with colleagues, the sputtering of frozen gases on planetary surfaces by energetic particles in the solar system. In this case, experiments showed very large sputtering rates that could only be explained by electronic excitation of the material being sputtered, a phenomenon limited in effect to insulators.

Most recently, Walter Brown has directed his interests to laser- solid interactions. It was he, together with colleagues, who first demonstrated that the annealing of implantation damage by $\mathrm{cw}$ laser irradiation is accomplished in the solid phase; i.e., does not involve melting.

Research interests aside, Walter Brown is known within the international community of scientists as a nurturer of colleagues, a friend and confidant of many, and as a professional of great integrity and vision. Brown is involved. He is involved with the members of his own research group, with his colleagues at Bell Laboratories, and with the world of science. Not insignificantly, Walter Brown is also involved with the Materials Research Society. His election to membership of the Council is announced in this issue of the BULLETIN, and his leadership role in the production of our Annual Meeting this year is apparent to all members of the Society who are able to attend.

\section{Walter Brown discusses Walter Brown}

Soon after coming to Bell Laboratories in 1950, fresh from a Ph.D. thesis with Purcell at Harvard, I worked with Bill Schockley in the new and rapidly growing field of semiconductors. It was all Germanium in those early days and I had a fascinating time studying surface states and surface inversion layers using field effect surface conduction and capacitance measurements. It was possible to determine the location and density of slow and fast surface states and the surface potential of $\mathrm{Ge}$, which could be strongly modified by the chemical environment.

The idea for field-effect-transistors already existed in Schockley's fertile mind, but we didn't have a good passive oxide. It was exciting to play a part in such a new area in a lab that had started it all. I also got interested in the damage introduced in germanium by high energy particles, primarily $\mathrm{MeV}$ electrons, and the way the damage annealed. These early efforts with R.C. Fletcher were to produce simple defects, and to sort them out by their positions in the energy gap with conductivity and Hall coefficient measurements, and with the kinetics of their interaction and annihilation. Access to highquality materials with a wide range of dopants and concentrations was critical to this work, and we were lucky to be in the middle of an enterprise where that was the name of the game.

In the late 50 s and early $60 \mathrm{~s} I$ became enamored with semiconductor-pn-junction particle detectors, because of nuclear physicists who had joined the department. This led to the "race for space" for me as Bell Labs made an enormous concentrated push on Telstar in the early 60s. Because of people like Burton, Buck and Miller, I had a chance to fly experiments on Telstars I and II, to measure the components of the high energy particle environment in space with detectors, and to examine their integrated effect through damage to semiconductor electronic components. Solar cells, exposed as they were to the environment, were devices of primary concern. We measured their degradation directly, and compared it with lab studies of the energy and particle dependence of defect formation. Electronically produced radiation effects in transistors also turned up in these experiments: one expected, and one not.

The first was, of course, the effect of hole-electron pairs produced by the radiation contributing currents in devices, as in semiconductor particle detectors. The second was modification of surface chemistry through ionization of species on the surface, and the modification of the junction properties as the ions moved under the influence of the junction potentials, a subject still fascinating. It was a fabulous time, when a large fraction of all of Bell Labs was caught up in this huge, important, and enormously successful Bell System project.

In the middle sixties, the subject of channeling of high energy particles in crystalline solids was independently discovered in England and in the U.S. by Dearnaly, Gibson, and Owen, and it fascinated me. I got involved in studies of channeling and dechanneling with Appleton and Feldman, and in the location of impurities and the measurement of disorder using this new technique.

Back to radiation damage-but now associated with ion implantation-and to analysis of implanted impurities in silicon. This interest extended into the seventies. Late in that decade, together with Poate, Leamy, Celler and many others at Bell Labs and elsewhere, I was caught up in the excitement of laser annealing, and solid and liquid phase regrowth of materials. I have been a fascinated kibitzer and critic in the evolution of this work to studies of rapid crystaliztion and impurity incorporation.

During this same period I became interested in sputtering, a rather mundane phenomenon in many ways, but with Mayer and Poate, not so mundane when examined and compared for alloy systems where preferential effects are rampant. And not so mundane either when the materials are insulators and the sputtering is created by electronic excitation rather than by momentum transfering collisions. These are newly discovered phenomenoa, not yet well understood. And this has led in the 80 s to interest, with Wagner, in ion beam modification of materials such as resists, and to finely focused ion beams and new high brightness ion sources, which can be used to modify materials near their surfaces in high resolution ways. 


\section{W.O. Baker discusses Walter Brown}

These notes on the achievments of Walter L. Brown were provided by William $O$. Baker, Chairman of the Board of Rockefeller University and a past receipient of the Von Hippel Award. Baker, as emeritus president of AT\&T Bell Laboratories, discusses Brown's contributions in the context of both Bell Laboratories and the world of science at large.

As Bell Laboratories moved into the full scale era of solid statesemiconductor-transistor science and technology that it had initiated just before and after World War II, it was desired to reorient and to stimulate new work in the classic and technologically productive fields of charged particles outside of matter. It was also decided coordinately to probe the significance of the rapidly developing sources of elementary particles from nuclear reactors and improved accelerators for their role in basic science, in telecommunications, in electronics, and in the solid state itself.

In this environ, Dr. Walter Brown joined the research area of Bell Laboratories at the end of 1950, upon completing his work in physics at Harvard. He soon

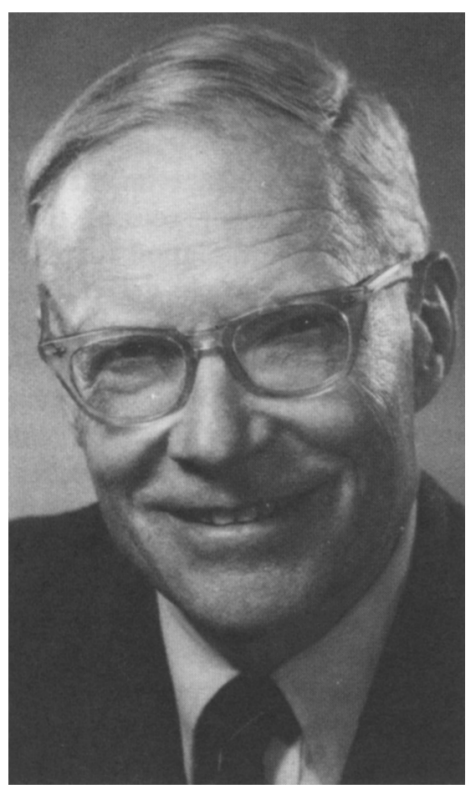

W.O. BAKER became a prime figure in the research strategy and findings related to the matters noted above. His early papers illuminated the effects of particle impact and radiation damage generally on junction devices and other solids. Coordinately, he showed how field effects as well as particle impacts (including photons) influenced transistor and other semiconductor surfaces. From these years in the mid and later 1950s, he has continued unabated to create enduring domains of knowledge about the interactions of elementary particles with crystals, especially those most sensitive to modification by charge and fields.

Thus, his work has been extraordinarily versatile, including the practical techniques of using semiconductor systems as particle detectors, and likewise revealing much of the structure and behavior of crystals by the effects of bombardment by both carefully controlled accelerator beams and by the particle fluxes of the cosmos. Walter Brown's development of this important junction of two major fields in 20th Century science and technology - the physics and materials properties of crystals and other solids, and the behavior of particle beams and collisions - has been characterized by excellent taste in choice of experiments and elegant instrumentation creation, coupled with keen appreciation of theory and the way it should be linked to observation. For instance, his findings about ion implantation and channeling laid the base for our fruitful and now universally applied methods for producing integrated circuitry. Here he complemented and extended the earliest observations at Harwell Laboratories in the United Kingdom.

The influence of Brown and his associates, with each of whom he has worked skillfully and carefully to encourage many independent careers, on the behavior of matter in outer space, has been preeminent in that domain. As we sought, following the President's Science Advisory Committee Space Primer of the late 50 's, to describe the conditions in both near-earth and far-out regions, Brown's radiation detectors have revealed unexpected qualities of the Van Allen Belts. Bombardment features of cosmic particles of ice and other solids have also been essential in understanding the radio transmission originated for satellites by the Telstar vehicle and its descendants.

These judgements of the wide-ranging significance of his work derive from our associations with it also through engineering and operational experience. The values of his studies apply similarly to effects in the innermost regions of crystals and surfaces. The photon bombardment research from laser treatment of surfaces has displayed typical expert insight and action. Walter Brown's demonstration of controlled conversion of texture on surfaces is of highest practical importance. As always, he has himself participated in the work and its interpretation so closely that he could constantly assess both external progress and the developing abilities of his colleagues and his laboratory department.

These remarkable qualities of skillful organization and group administration combined with keen individual talents for discovery and application are in the highest tradition of the Bell Laboratories community. Accordingly, Walter Brown was repeatedly offered appointments expanding his leadership responsibilities, which in each case he pondered carefully, but chose instead to follow devotedly the important pathways of personal involvement in dynamic and technologically potent fields of science. Nevertheless, his own large and vigorous research group was always encouraged to work on the frontier, in new directions. The superb families of apparatus for the study of particles colliding with crystals is a worthy sequence to the probings of Davisson and Germer in finding the wave nature of the electron, the experimental expression of quantum mechanics.

As suggested above, Walter Brown not only moved strongly and recruited effectively to establish new programs for interaction of solid state and particle physics, but he also has kept essential connections between our total Bell Laboratories research and the vast new domains of nuclear physics.

Far from being oppressed by these large missions and responsibilities, Walter has unswervingly maintained personal enthusiasm and charisma for research. His worldwide links, such as with institutes in Denmark, and the succession of fine visiting scholars to his department are further accents of his achievements in bonding physics and the humanities. 


\section{Previous Von Hippel Winners}

The first recipient of the award was the man whose name it bears, Arthur Robert von Hippel, whose pioneering research and unfettered spirit inspired the prize. Emeritus professor of the Massachusetts Institute of Technology, von Hippel's research in dielectrics, semiconductors, ferromagnetics and ferroelectrics resulted in the publication of two visionary books, Molecular Science and Molecular Engineering and Molecular Designing of Materials and Devices.

Von Hippel studied electrophysics at the University of Goettingen, which granted him the Ph.D. in 1924. After a decade of teaching and research in Europe he joined the MIT faculty in 1936. It was in his lab that the example of scientists working cooperatively to solve the mysteries of materials from the atomic to the microstructural level first challenged the parochialism that had prevailed before and demonstrated the utility of the interdisciplinary approach which the Materials Research Society hopes to foster and embody.

The second recipient of the prize, in 1978, was W.O. Baker, president of Bell Telephone Laboratories. Baker joined Bell Labs in i939, having received his Ph.D. in physical chemistry from Princeton the previous year. First as a member of the technical staff and subsequently in various leadership positions, he studied solid state materials and macromolecules, dielectric and dynamic mechanical properties of crystals and glasses, information processing technology, and plastics, fibers and natural and synthetic rubbers.

In addition to his research, Baker has devoted himself to numerous civic, governmental and scientific committees and commissions. In 1957 Washington University, where he had done his undergraduate work, awarded him its honorary Doctor of Science degree. At least seventeen other institutions of higher education have brought honor to themselves with similar awards.

David Turnbull, Gordon McKay Professor of Applied Physics at Harvard, was the Von Hippel Award recipient in 1979. A physical chemist by training, Turnbull's research has encompassed a broad range: thermionic emission, thermodynamic properties of gases at high pressures, corrosion in non-aqueous media, diffusion in metals, kinetics of nucleation in solid state transformation, solidification, theory of liquid and glass.

Turnbull received his Ph.D. from the University of Illinois in 1939. He began his career at Case Institute of Technology, then joined the research laboratory of the General Electric Company in 1946, where he remained until he joined the Harvard faculty in 1962.

W. Conyers Herring, 1980 recipient of the prize, is professor of applied physics at Stanford University. He has had a seminal influence on materials science and solid state physics, contributing to the understanding of solid surfaces that underpins the fields of crystal growth, sintering and plastic flow at high temperatures. Together with J.K. Galt he realized and demonstrated that whiskers of high crystalline perfection would exhibit extraordinary mechanical properties.
Herring received his Ph.D. from Princeton in physics in 1937. He taught at MIT, Princeton and the University of Missouri, and from 1941 to 1945 was a member of the War Research staff at Columbia University. After 30 years at Bell Laboratories, he joined Stanford in 1976.

James W. Mayer, the Society's 1981 honoree, is Francis Norwood Bard Professor of Materials Science and Engineering at Cornell University. Mayer has had a profound influence on the development of modern materials science with particular emphasis on semiconductor materials. Almost every area he has worked in has turned out to be important. For example, his research on implantation identified the damage and epitaxial regrowth phenomena long before the technique was accepted as an integral part of the semiconductor industry. He has been a pioneer in the use of ion beam techniques for materials analysis.

Mayer received his Ph.D. in physics from Purdue in 1960. He was with Hughes Aircraft Company until 1967, when he became professor of electrical engineering at Caltech, where he remained until joining Cornell in 1980. Among his other accomplishments at Caltech, he was a scuba-diving instructor for a decade.

Clarence M. Zener, emeritus university professor of CarnegieMellon, received the Von Hippel Award in 1982. His contributions to the physics of metals and to mathematics are among the most fundamental and original of any 20th-century scientist. He performed the definitive work on internal friction in solids, and provided the theoretical foundation for a line of research that resulted in the invention of the Zener diode and may be said to underlie the development of semiconductors.

A graduate of Harvard, which granted him the Ph.D. in 1929, Zener accepted a series of academic appointments, interrupted by a 15-year association with Westinghouse Electric Company, during which he built a research institution among the finest in the world. He was appointed university professor at Carnegie-Mellon in 1968.

The Von Hippel Award in 1983 was presented to Sir Peter B. Hirsch, Isaac Wolfson Professor of Metallurgy at the University of Oxford and Chairman of the United Kingdom Atomic Energy Authority. The first person to observe dislocations in transmission electron microscopy, Sir Peter is the author, with others, of the fundamental text in the field, Electron Microscopy of Thin Crystals. His The Physics of Metals, Vol. II, Defects, is equally important. With his colleagues and students, Sir Peter is largely responsible for the elevation of electron microscopy to its preeminent position in the study of the microstructure of materials.

Sir Peter took his Ph.D. at Cambridge in 1951. He pursued an academic career, culminating in his appointment to Oxford in 1966 as head of the Department of Metallurgy and Science of Materials. A Fellow of the Royal Society and a recipient of its Royal Medal, Sir Peter has received scores of prizes and honorary degrees, notably the Rosenhain Medal of the Institute of Metals, and the C.V. Boyes Prize of the Institute of Physics and Physical Society. He was made Knight Bachelor in 1975. 